\title{
Adjuncts to local anaesthetics in total knee arthroplasty
}

\section{Commentary}

We recently read with interest 'Efficacy of local infiltration analgesia in total knee arthroplasty: A randomised, placebo-controlled, double-blind trial' by Unver and colleagues. ${ }^{1}$ Post-operative pain is a common source of patient dissatisfaction. ${ }^{2}$. The results of this study showed a significant reduction in post-operative pain and better recovery of post-operative knee flexion. This highlights the often under-appreciated role of local infiltration analgesia (LIA) in the management of early post-operative pain. In 2016 Albrecht et al. ${ }^{3}$ published a meta-analysis of 1,112 patients sourced from fourteen randomised controlled trials, comparing LIA with femoral nerve block (FNB) in total knee arthroplasty (TKA). ${ }^{3}$ This study showed LIA to be equivalent to FNB in regard to 24 hour post-operative pain scores, morphine consumption and complications such as infection and falls. The LIA group received significantly higher local anaesthetic doses, however this did not result in elevated plasma concentrations or systemic toxicity. The authors of this study hypothesised that LIA may actually be preferable to FNB due to the ease and availability of LIA, as well as a theoretical reduction in the incidence of motor blockade.

Local anaesthetic adjuncts are a growing field of acute pain management and may provide additional benefits when LIA is used for TKA. A recent systematic review by Kirksy et al. ${ }^{4}$ demonstrated a consistent benefit associated with the use of agents such as clonidine, dexmedetomidine, magnesium, and dexamethasone to increase the efficacy of peripheral nerve blockade (PNB) compared to local anaesthetic alone. ${ }^{4}$ Meta-analyses investigating the addition of alpha-agonists (clonidine ${ }^{5}$ and dexmedetomidine) ${ }^{6}$ to PNB have been shown to increase the duration of sensory blockade (122 and 284 minutes respectively) and thus reduce post-operative pain. The utility of these agents may be limited by their systemic side effects such as bradycardia and hypotension. Magnesium has been shown to significantly augment PNB by 106 minutes without any significant systemic side effects. ${ }^{7}$ Similarly, the addition of dexamethasone showed a significant increase in sensory blockade by 488 minutes, also without systemic side effects. ${ }^{8}$ All of the meta-analyses showed an undesirable increase in the duration of motor blockade, a side effect avoided with LIA. LA additives have also been proven effective in LIA during tonsillectomy. A meta-analysis by Vlok et al. ${ }^{9}$ showed that agents such as dexamethasone, magnesium, pethidine and tramadol significantly reduce post-operative pain and analgesia requirements post-tonsillectomy. ${ }^{9,10}$ There was no associated increase in systemic side effects.

To date there is a paucity of literature looking specifically at the utility of adjuncts to LIA in TKA. Given the benefit demonstrated in PNB and tonsillectomy, investigation of LIA adjuncts in TKA is warranted.

\section{Acknowledgements}

None.

\section{Conflicts of interest}

The authors declare that there is no conflict of interest.
Volume 7 Issue 6 - 2017

\author{
Matthew Binks,' Elyse Bond, ${ }^{2}$ Thomas Ryan, ${ }^{3}$ \\ Ruan Vlok, ${ }^{4}$ Thomas Melhuish, ${ }^{5}$ Leigh White ${ }^{6}$ \\ 'Wagga Wagga Rural Referral Hospital, Australia \\ 2University of New South Wales, Australia \\ ${ }^{3}$ Caboolture Hospital, Australia \\ ${ }^{4}$ University of Notre Dame, Australia \\ 5John Hunter Hospital, Australia \\ UUniversity of Wollongong, Australia
}

Correspondence: Leigh White, University of New South

Wales, University of Wollongong, Australia,

Email lw844@uowmail.edu.au

Received: April 25, 2017 | Published: April 25, 2017

\section{References}

1. Unver B, Yuksel E, Kalkan S, et al. FRI0620-HPR Efficacy of Local Infiltration Analgesia in Total Knee Arthroplasty: A Randomised, Placebo-Controlled, Double-Blind Trial. Annals of the Rheumatic Diseases. 2016;75:1278.

2. White L, Holyoak R, Sant J, et al. The effect of infrapatellar fat pad resection on outcomes posttotal knee arthroplasty: a systematic review. Arch Orthop Trauma Surg. 2016;136(5):701-708.

3. Albrecht E, Guyen O, Jacot-Guillarmod A, et al. The analgesic efficacy of local infiltration analgesia vs femoral nerve block after total knee arthroplasty: A systematic review and meta-analysis. BJA. 2016;116(5):597-609.

4. Kirksey MA, Haskins SC, Cheng J, et al. Local anesthetic peripheral nerve block adjuvants for prolongation of analgesia: a systematic qualitative review. PloS one. 2015;10(9):e0137312.

5. Pöpping DM, Elia N, Marret E, et al. Clonidine as an Adjuvant to Local Anesthetics for Peripheral Nerve and Plexus BlocksA Meta-analysis of Randomized Trials. Anesthesiology. 2009;111(2):406-415.

6. Abdallah FW, Brull R. Facilitatory effects of perineural dexmedetomidine on neuraxial and peripheral nerve block: a systematic review and metaanalysis. Br J Anaesth. 2013;110(6):915-925.

7. Li M, Jin S, Zhao X, et al. Does Magnesium Sulfate as an Adjuvant of Local Anesthetics Facilitate Better Effect of Perineural Nerve Blocks?: A Meta-analysis of Randomized Controlled Trials. Clin J Pain. 2016;32(12):1053-1061.

8. Albrecht E, Kern C, Kirkham KR. A systematic review and meta-analysis of perineural dexamethasone for peripheral nerve blocks. Anaesthesia. 2015;70(1):71-83.

9. Vlok R, Melhuish TM, Chong C, et al. Adjuncts to local anaesthetics in tonsillectomy: a systematic review and meta-analysis. J Anesth. 2017.

10. Vlok R, Borowczyk K, Melhuish TM, et al. Magnesium as an adjunct to local anaesthetic prolongs postoperative analgesia and decreases laryngospasm in tonsillectomy. J Anesth Pain Med. 2016;1(2):1-2. 